\title{
Medizinische Ergebnisqualität: Ärztlicher Auftrag und Managementaufgabe
}

\author{
M. Heberer ${ }^{1}$ \\ A. Prengel 1
}

Qualitätsmanagement

Schlüsselwörter

Qualitätsmanagement

Ergebnisqualität

Organisationsstruktur

Key words

quality management

medical outcome

organizational structure

Institut

${ }^{1}$ Institut für Chirurgische Forschung und Spitalmanagement (ICFS), Forschungsgruppe Spitalmanagement,

Universitätsspital Base

Bibliografie

DOI $10.1055 / \mathrm{s}-0028-1085588$

Dtsch Med Wochenschr 2008;

133: S144 - (c) Georg Thieme

Verlag KG Stuttgart - New York ISSN 0012-0472

Korrespondenz

Prof. Dr. med. Michael Heberer, M.B.A.

Leiter Institut für Chirurgische Forschung und

Spitalmanagement (ICFS)

Mitglied der Spitalleitung

Universitätsspital Basel

Hebelstr. 20

$\mathrm{CH}-4031$ Basel

Tel. +41 (0) 612652375

eMailmheberer@uhbs.ch
Struktur-, Prozess- und Ergebnisqualitäten sind wertbestimmende Merkmale von Gesundheitsleistungen. Die medizinische Ergebnisqualität nimmt einen besonderen Stellenwert ein, weil es sich um die „raison d'être“ der Medizin handelt. Allerdings ist die Ergebnisqualität von Laien und Professionals oft schwer beurteilbar, weil die angestrebten Ergebnisse unzureichend definiert sind und die Zielerreichung somit nicht messbar ist. Dennoch bleibt die kontinuierliche Verbesserung der medizinischen Ergebnisqualität ein allgemein akzeptiertes Ziel.

\section{Qualität als ärztliche Kernaufgabe} $\nabla$

Die Entwicklung der Medizin in den Industrieländern hat Krankheiten beseitigt, Lebenszeiten verlängert und Möglichkeiten zur Linderung von Leiden geschaffen. Ärzte haben die zugrunde liegenden Innovationen erarbeitet, Kriterien zur Bewertung von medizinischen Verfahren geschaffen (evidence based medicine) und die Erkenntnisse verbreitet. Das spricht für einen ärztlichen Beitrag zur Steigerung der Behandlungsqualität. Auch weiterhin wird die Förderung der Qualität medizinischer Leistungen eine unbestrittene Kernaufgabe von Ärzteschaft und Pflege sein.

\section{Qualitåt als Differenzierungsmerkmal $\nabla$}

Unter den Bedingungen von Festpreisen (DRG, TARMED etc.) können medizinische Leistungen nur in Hinblick auf erwartete und wahrgenommene Qualitätsvorteile ausgewählt werden. Im resultierenden Qualitätswettbewerb der Leistungsanbieter können Ergebnisqualitäten ebenso wie Struktur- und Servicemerkmale in den Vordergrund gestellt werden: Je mehr medizinische Leistungen standardisiert und/oder Differenzierungen der Ergebnisqualität für den Patienten nicht wahrnehmbar sind, desto mehr werden Servicequalitäten zur Differenzierung herangezogen.

\section{Qualität als Managementaufgabe}

Die Entwicklung der medizinischen Leistungsfähigkeit ist mit der medizinischen Spezialisierung untrennbar verbunden. In Großkliniken sind heute mehr als 60fachliche Einheiten abgrenzbar. Diese feinkörnige Segmentierung der Medizin hat dazu geführt, dass der typische Patient Leistungen aus mehreren Einheiten in Anspruch nimmt. Zum einen resultieren Schnittstellen hinsichtlich Organisation und Information, deren Koordination von den medizinischen Spezialisten kaum bewältigt werden kann. Zum anderen entstehen Risiken, dass nicht alle medizinischen Spezialgebiete ihre Infrastruktur auslasten und qualitätsrelevante Mindestfallzahlen erreichen können. Der hohe Spezialisierungsgrad stellt somit qualitätsrelevante Anforderungen an das Management.

\section{Konzepte des Qualitätsmanagements}

Qualitätsmanagement ist eine kontinuierliche und zielorientierte Aufgabe. $\mathrm{Zu}$ jedem Zeitpunkt müssen konkrete Ziele des Qualitätsmanagements festgelegt werden. Im aktuellen Umfeld wird folgenden Zielen grundsätzliche Bedeutung zugesprochen:

(i) Lateralisierung: Die prozessuale Koordination über Organisationseinheiten erfordert eine Lateralisierung der traditionell funktional-vertikalen Organisationsstruktur. Die Entwicklung von Behandlungszentren, in denen jeweils mehrere Spezialgebiete koordiniert werden, wird als Beispiel beschrieben.

(ii) Transparenz von Leistungen und Ergebnissen: Die Offenlegung von Leistungsgeschehen und Ergebnissen wird in der Medizin notwendig, wenn man mündigen Patienten Wahlmöglichkeiten einräumen möchte. Dazu werden das Konzept der Qualitätssicherung über Routinedaten beschrieben und die Notwendigkeit zur Weiterentwicklung abgeleitet (vgl. iii).

(iii) Qualitätsziele: In Hinblick auf den Qualitätswettbewerb sind konkrete betriebliche Qualitätsziele zu definieren, um Wettbewerbsvorteile zu erarbeiten und den Bestand des Unternehmens zu sichern. Beispiele aus dem eigenen Spital werden zur Diskussion gestellt.

Zusammenfassend ist die kontinuierliche Verbesserung der Ergebnisqualität im Gesundheitswesen nur durch das Zusammenwirken von allen Berufsgruppen zu erreichen. Diese sind zudem auf die Mitwirkung der Patientinnen und Patienten angewiesen, die deshalb auch als Koproduzenten der Dienstleistung bezeichnet werden. Damit wird die Verbesserung der Ergebnisqualität zu einer integralen Aufgabe, an deren Realisierung alle Beteiligten Verantwortung und Anteil haben.

Autorenerklärung: Die Autoren erklären, dass keine relevanten finanziellen Verbindungen in Bezug auf dieses Manuskript bestehen. 